\title{
CARACTERIZACIÓN DE LA COBERTURA ARBÓREA EN UNA PASTURA DEL TRÓPICO SECO EN TOLIMA, COLOMBIA ${ }^{1}$
}

\author{
Jorge Rodrigo Serrano ${ }^{2}$, Hernán J. Andrade C. ${ }^{3}$, Jairo Mora-Delgado ${ }^{4}$
}

\begin{abstract}
RESUMEN
Caracterización de la cobertura arbórea en una pastura del trópico seco en Tolima, Colombia. El objetivo de este estudio fue caracterizar la vegetación leñosa perenne de un potrero arbolado del Magdalena Tolimense en Colombia. El inventario forestal se realizó mediante el establecimiento de parcelas temporales de muestreo. La estimación del porcentaje de oclusión de la copa de los árboles se obtuvo mediante fotografías digitales al cenit y su análisis en el software libre Gap Light Analyzer. La radicación fotosintéticamente activa (RAFA) transmitida por la copa se midió con un ceptómetro Accupar LP-80. Se encontraron 21 especies leñosas perennes en un área de muestreo de 54 ha. Con menor porcentaje de oclusión fueron: Pseudosamanea guachapele (64\%) y Prosopis juliflora (63\%), lo que sugiere incluirlas en diseños de sistemas silvopastoriles; algunas especies poseen potencial maderable como ingreso adicional al ganadero. Se encontró una importante diversidad de especies leñosas para ser introducidas en pasturas, sin perjudicar la producción de materia seca comestible para el ganado.
\end{abstract}

Palabras claves: ganadería, inventario forestal, sistemas silvopastoriles, transmisión de RAFA.

\begin{abstract}
Characterization of tree cover in a dry tropical pasture in Tolima, Colombia. The objective of this study was to characterize the woody perennial vegetation of a pasture from Tolima's Magdalena valley in Colombia. The forest inventory was carried out by establishing temporary sampling plots. The estimation of canopy occlusion was obtained through digital photographs of tree crowns taken near sunset and were analyzed with Gap Light Analyzer software. Transmitted Photosynthetic Active Radiation (PAR) was estimated with an Accupar ceptometer LP80. A total of 21 woody perennial species was found in a sampling area of 54 ha. Pseudosamanea guachapele (64\%) and Prosopis juliflora (63\%) were the tree species with the lowest occlusion, suggesting its potential use in silvopastoral systems; some timber species have potential as additional income to the farmer: A significant diversity of woody perennial species with potential to be introduced into pastures, without affecting the production of edible dry matter for livestock were found.
\end{abstract}

Keywords: tropical dry forest livestock, forest inventory, transmitted PAR, silvopastoral systems.

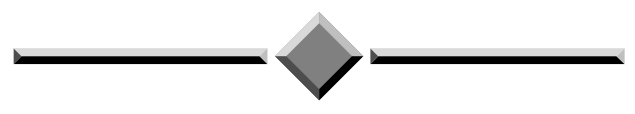

\footnotetext{
Recibido: 24 de julio, 2013. Aceptado: 18 de marzo, 2014. Parte de la tesis de Maestría en Ciencias Agrarias del primer autor. "Interacción entre cobertura arbórea y comportamiento animal durante las épocas seca y húmeda en pasturas del Magdalena Medio Tolimense". Convenio Universidad del Tolima-Universidad Nacional de Colombia, Palmira.

2 Centro Universitario Regional del Norte (CURDN), Universidad del Tolima, Colombia. rodrigose1000@hotmail.com

3 Grupo de Investigación Producción Ecoamigable de Cultivos Tropicales (PROECUT), Universidad del Tolima, Colombia. hjandrade@ut.edu.co

4 Grupo de Investigación Sistemas Agroforestales Pecuarios (SAFP), Universidad del Tolima, Colombia. jrmora@ut.edu.co
} 


\section{INTRODUCCIÓN}

Los países tropicales se caracterizan por alta precipitación, humedad relativa, temperatura y radiación solar, lo cual genera condiciones ambientales heterogéneas en agroecosistemas con alta diversidad en oferta forrajera para alimentación animal a partir de especies leñosas y herbáceas. Dependiendo de la disponibilidad, disposición e interacción entre el componente arbóreo y herbáceo, el ganado puede favorecerse por la oferta forrajera, especialmente en la época seca y mejorar la eficiencia zootécnica, al reducir el estrés calórico en zonas de bosque seco tropical. Al igual, el suelo puede ser beneficiado por el incremento de la fertilidad (Belsky et al., 1993). Aproximadamente el $50 \%$ de las zonas dedicadas a pastoreo en Colombia presentan algún nivel de degradación, situación que obliga a desarrollar estudios en renovación y rehabilitación de praderas, implementando nuevos diseños y modelos, donde los sistemas silvopastoriles juegan un papel importante (Chamorro et al., 1998).

Después de considerar los potreros arbolados como campos enmalezados y "no aptos" para la producción ganadera, los sistemas agroforestales pecuarios empiezan a generar otro enfoque válido y sostenible como opción de producción pecuaria. Así, en algunas fincas con tradición de uso de potreros sin árboles, se están estableciendo leñosas perennes en las pasturas con el fin de producir otros bienes y servicios diferentes a los pecuarios, principalmente madera, fibras y frutos, lo que genera un sistema de producción sustentable con muchos beneficios ambientales (González-Hernández y Rozados-Lorenzo, 2008; Harvey et al., 2005). Uno de los sistemas agroforestales más frecuentes y dominantes en Colombia son los árboles dispersos en potreros.

El componente leñoso en pasturas cumple un papel muy importante en la productividad de las fincas ganaderas ya que proveen múltiples bienes, como la madera y alimento para el ganado y servicios como la sombra, la fijación de carbono y la conservación de la biodiversidad (Harvey y Haber, 1999; Zamora et al., 2001; Andrade et al., 2008). Algunos estudios han demostrado el impacto positivo de los árboles en la producción ganadera, por ejemplo, Souza de Abreu (2002) encontró que la presencia de sombra de árboles ha provocado incrementos de alrededor de un $13 \%$ en la producción de leche en la zona del trópico húmedo de Costa Rica.
Los árboles en pasturas son capaces de brindar beneficios ecológicos, como la contribución a conservar hábitats para especies animales, importantes en la dispersión de semillas arbóreas para la regeneración natural, y funcionan como corredores biológicos (Harvey y Haber, 1999). Además, las especies leñosas pueden contribuir a la restauración de pasturas degradadas (Szott et al., 2000), como sumideros de carbono (Andrade et al., 2008) y como alternativa estratégica para reducir la presión sobre los bosques (Kaimowitz y Angelsen, 2008).

Algunos estudios en fincas ganaderas del trópico húmedo de Costa Rica, reportan una densidad de árboles dispersos en pasturas de 12 a 22 árboles/ha (Souza de Abreu et al., 2000), mientras que en el trópico seco de Nicaragua se registraron densidades de 14 a 82 árboles/ha (Zamora et al., 2001). Por otro lado, los conocidos efectos devastadores de la ganadería tradicional sobre el medio ambiente, producto de la tala y quema indiscriminada, se ha generado la necesidad de implementar modelos que reduzcan el impacto negativo sobre los recursos naturales como los sistemas agroforestales, en los que se pretende conservar el componente arbóreo y el aprovechamiento de la diversidad de recursos forrajeros presentes en el medio. Ospina (2003) hace un recuento de las experiencias más favorables de árboles dispersos en potreros de Colombia, encontrándose una gran diversidad de especies que pueden ser utilizadas en sistemas de producción animal. La ganadería predominante en el valle cálido del alto del Magdalena se ha orientado a la producción de carne aprovechando pasturas degradadas y gramas naturales, las cuales se encuentran frecuentemente asociadas a especies arbóreas, aunque con un deficiente manejo y aprovechamiento (Andrade et al., 2010).

El objetivo de este estudio fue caracterizar la vegetación leñosa perenne de un potrero arbolado del Magdalena Tolimense en Colombia.

\section{MATERIALES Y MÉTODOS}

\section{Área de estudio}

El estudio se realizó entre agosto de 2009 y agosto de 2010 en la hacienda Pajonales, perteneciente a la Organización Pajonales S.A., localizada en el municipio de Ambalema, Tolima, Colombia, parte alta de la cuenca del Magdalena. El sitio de estudio consistió en 
54 ha localizadas entre las coordenadas $4^{\circ} 45^{\prime} 25,9^{\prime \prime} \mathrm{N}$ y $74^{\circ} 52^{\prime} 20,8^{\prime \prime} \mathrm{O}$ a una distancia de $15 \mathrm{~km}$ del casco urbano del municipio de Ambalema (Figura 1). La zona presenta una precipitación media anual de 1270 $\mathrm{mm}$, una temperatura media de $28^{\circ} \mathrm{C}$ y una altitud de $300 \mathrm{~m}$, ubicándose en una zona de vida de bosque seco tropical (Holdridge, 1967). La mayor parte de los suelos corresponden a vertisoles, inceptisoles y molisoles. Los sistemas productivos de la hacienda Pajonales están dirigidos a diversas actividades de tipo agropecuario, entre ellas la ganadería.

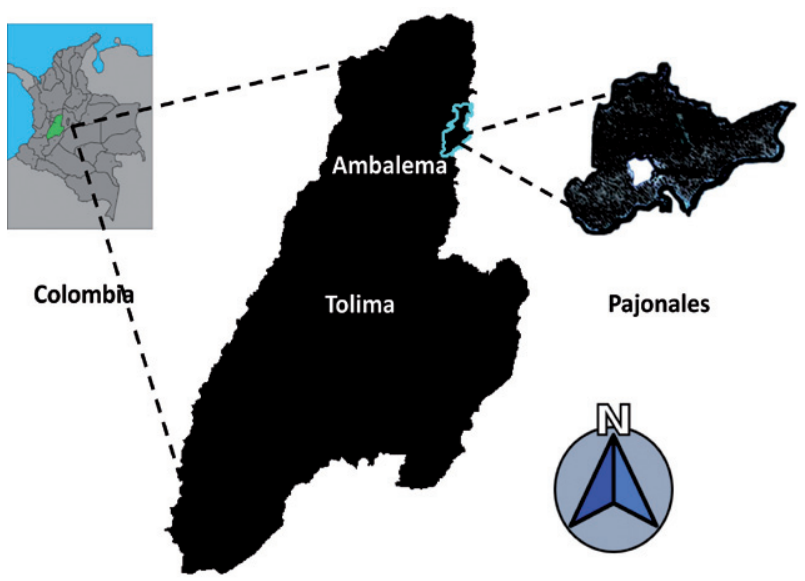

Figura 1. Localización del área de estudio en la Hacienda Pajonales, Ambalema, valle cálido del Magdalena Tolimense, Colombia. 2013.

Inventario forestal y estimación de cobertura arbórea

El inventario forestal se estimó en un potrero de 54 ha (Figura 2) con una topografía que varía entre plana y ondulada. El área de estudio se caracteriza por tener una variada cobertura arbórea que va desde 0 a $60 \%$. El potrero en estudio se delimitó mediante georeferenciación con un GPS submétrico, además, se contó con una delimitación física (cercas).

El inventario forestal se realizó mediante el establecimiento de parcelas temporales de muestreo, de forma y tamaño variable localizadas en función de la distribución espacial del componente leñoso estimado visualmente. En estas parcelas se inventariaron las

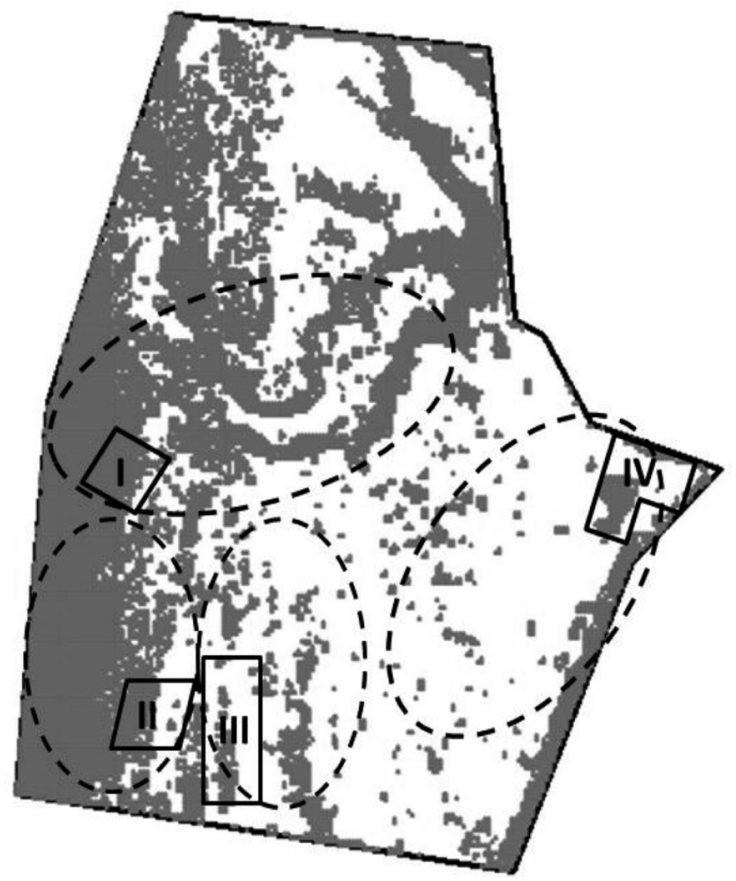

Figura 2. Ubicación espacial de parcelas en el potrero de estudio representadas mediante óvalos, para la caracterización del componente leñoso perenne en pasturas del Magdalena Tolimense, Colombia. 2013. Las líneas negras corresponden a las parcelas y líneas punteadas de los óvalos indican el área homogénea que representa cada parcela (2013).

especies leñosas y se ubicaron geográficamente por medio de un receptor de GPS (Garmin eTrex Vista). Cuando la especie no era identificada en el campo, se procedió a recolectar muestras de hojas, flores y frutos para una posterior identificación taxonómica en el Herbario Toli de la Universidad del Tolima.

La evaluación de la cobertura arbórea se realizó en la época lluviosa, octubre a diciembre de 2009. Se delimitaron cuatro parcelas en el potrero de diferente tamaño, así: I y II de $7000 \mathrm{~m}^{2}$ cada una, parcela III de $15000 \mathrm{~m}^{2}$ y IV de $12000 \mathrm{~m}^{2}$. Estas eran representativas de la distribución del componente leñoso de cada una de las áreas diferenciadas por estimación visual según la densidad arbórea (Figura 2).

\section{Estimación de parámetros dasométricos}

Se calculó la abundancia relativa (\%) de especies leñosas, área de copa $\left(\mathrm{m}^{2}\right)$, altura de los árboles (m) y 
se estimó el índice de valor de importancia (IVI). La altura total y de fuste de los árboles se estimó usando un clinómetro. El área de la copa se estimó midiendo dos diámetros perpendiculares de la proyección de la copa sobre el suelo con cinta métrica; se asumió que el área de proyección vertical es de forma elipsoide (Bellow, 2000). Se aplicó la fórmula $A=\pi / 4$ (diámetro promedio $^{2}$ ). El IVI se estimó como la sumatoria de la abundancia, dominancia y frecuencias relativas.

La abundancia relativa es la proporción porcentual de los individuos de cada especie entre el número total de los árboles. La frecuencia relativa es el cociente entre el número de parcelas en el que se encontró una especie y la frecuencia total de todas estas por 100. La dominancia relativa se calcula como la proporción porcentual del área basal de unas especies respecto al área basal total del sistema.

Simultáneamente a la realización del inventario forestal, se tomaron otras variables dasométricas de las leñosas perennes, tal como el diámetro del tronco a la altura del pecho (a 1,3 m de altura, DAP) y diámetro del tronco a la base (DAB).

\section{Oclusión de la copa}

Se tomaron fotografías digitales verticales desde abajo hacia arriba del dosel de las diferentes especies arbóreas del potrero, en las primeras horas de la mañana para evitar interferencia de rayos solares. Las imágenes fueron analizadas usando software libre Gap Light Analyzer, el cual fue diseñado para analizar fotografías hemisféricas. Esta herramienta permitió estimar el porcentaje de oclusión y la transmisión de radiación fotosintéticamente activa (RAFA) a través del dosel de las leñosas perennes (Frazer et al., 1999).

\section{Medición de transmisión de radiación fotosintética- mente activa (RAFA)}

La RAFA se midió con un ceptómetro AccuparLP-8, en cuatro posiciones: junto al tronco, en la parte media del dosel, en la frontera del dosel y fuera del dosel de la copa de los árboles, tomando diez mediciones en cada una de ellas. Además, la medición fuera de la copa, fue considerada como la RAFA incidente. Las mediciones se realizaron a medio día (entre 11:00 y 13:00) con cielo completamente despejado. La transmisión de RAFA se estimó mediante la siguiente ecuación:

$$
R A F A_{t(\%)}=\frac{R A F A t}{R A F A i} \times 100
$$

Donde,

RAFA $_{\mathrm{t}(\%)}:$ RAFA transmitida (\%)

RAFAt: RAFA transmitida (bajo copa, $\mu$ moles $\left.\mathrm{m}^{-2} \mathrm{~s}^{-1}\right)$

RAFAi: RAFA incidente (fuera de la copa, $\mu$ moles $\mathrm{m}^{-2} \mathrm{~s}^{-1}$ )

\section{Sombra en el potrero}

La sombra por potrero se estimó aplicando la siguiente fórmula:

$$
\text { Sombra }=\frac{\sum(\text { Aci } \times O i)}{\text { Atotal }} \times 100
$$

Donde,

Aci $=$ Área de copa del árbol $\left(\mathrm{m}^{2}\right)$

Oi = Oclusión del árbol

Atotal $=$ Área total del potrero $\left(\mathrm{m}^{2}\right)$

Se calculó la relación entre el área basal y el área de sombra de cada especie (Ab/As) como un índice del potencial de producción de madera en función de la sombra generada a las pasturas.

\section{Análisis estadístico}

Se realizó un análisis de estadística descriptiva y una prueba de Chi Cuadrado para estudiar si se presentan diferencias significativas en la abundancia relativa entre especies. Los análisis estadísticos de la cobertura arbórea en un potrero arbolado del trópico seco del alto del Magdalena se hicieron con el paquete estadístico SPSS v. 20.

\section{RESULTADOS Y DISCUSIÓN}

\section{Inventario forestal y estimación de coberturas}

El inventario de leñosas perennes reunió 21 especies correspondientes a igual número de géneros. 
Más del $81 \%$ de los individuos leñosos perennes cuantificados pertenecen a cinco especies: diomate (Astronium graveolens), guácimo (Guazuma ulmifolia), baho (Platymiscium hebestachyum), angarillo (Chloroleucon bogotense) y palma de vino (Sheelea magdalenensis), con 26, 25, 12, 11 y $8 \%$ de abundancia relativa, respectivamente (Figura 3 ). Se encontraron diferencias significativas $(\mathrm{p}<0,01)$ en la prueba de Chi Cuadrado para la abundancia relativa entre las especies en las parcelas de muestreo. Estos resultados concuerdan ampliamente con lo reportado por IAVH (1998) y Esquivel et al. (2003) para A. graveolens y G. ulmifolia, respectivamente, las cuales son definidas como abundantes y frecuentes en zonas de bosque seco tropical.

Las especies leñosas perennes de la región son usadas básicamente para suministrar sombra al ganado y algunas son utilizadas como maderables para poste de cerca y como aporte en la dieta de los bovinos. De las predominantes en el potrero de estudio, G. ulmifolia ofrece follaje y semilla como opción alimenticia muy apetecida por los vacunos y equinos, principalmente en épocas de escasez de forraje (ManríquezMendoza et al., 2011). A. graveolens es una especie con madera de alta resistencia a golpes, resistencia al rajado, buenas características de maquinado, lijado y dureza lateral alta, que la convierten en especie de uso maderable (Bárcenas-Pazos, 1995).

La cobertura arbórea varió entre el 23,4 y 77,4\% a nivel de parcelas. Sin embargo, se encontró una alta heterogeneidad de la sombra en cada cuadrante, presentando un porcentaje de cobertura entre 5 y $35,2 \%$ (Cuadro 1).

\section{Mediciones dasométricas}

El DAP para G. ulmifolia varió entre 15 y $70 \mathrm{~cm}$, lo cual está dentro del valor reportado por ZapataArango (2010) en los Municipios de Muy Muy y Matiguás, Departamento de Matagalpa, Nicaragua (promedio de $35,1 \mathrm{~cm}$ ). La especie A. graveolens presentó un valor promedio para DAP menor a $60 \mathrm{~cm}$, lo cual indica que hay muchos individuos pequeños, probablemente jóvenes (Arends et al., 2005); es decir, existe alta capacidad de regeneración natural de esta especie. La dominancia relativa muestra que el $78 \%$ del área basal corresponde a cinco especies: 25,3\% guácimo (Guazuma ulmifolia), 15,8\% diomate (Astronium graveolens), 16,4\% angarillo (Chloroleucon bogotense), 3,9\% baho (Platymiscium hebestachyum)

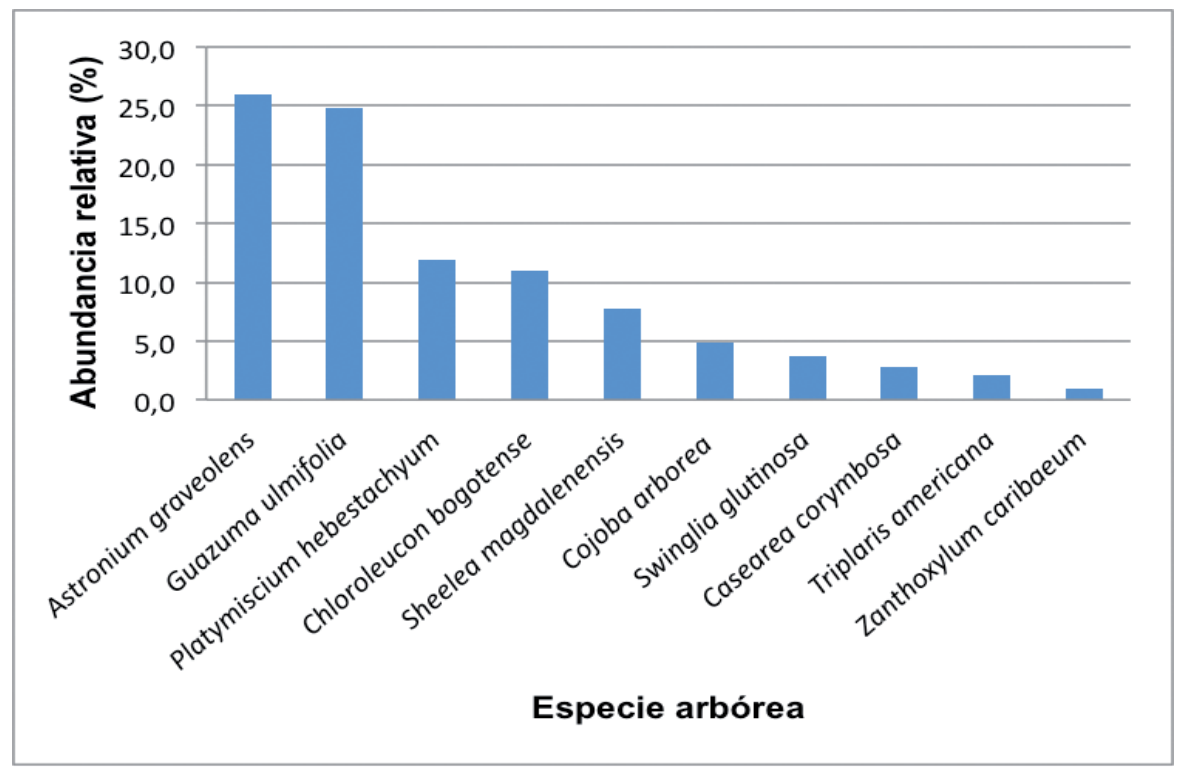

Figura 3. Abundancia relativa de especies leñosas del bosque seco tropical registradas en una pastura arbolada del Magdalena Tolimense, Colombia. 2013. 
Cuadro 1. Cobertura arbórea determinada en parcelas de monitoreo en una pastura arbolada del Magdalena Tolimense, Colombia. 2013.

\begin{tabular}{lcc}
\hline Cuadrante & $\begin{array}{c}\text { Abundancia abso- } \\
\text { luta (individuos/ha) }\end{array}$ & $\begin{array}{c}\text { Cobertura arbórea } \\
\text { en parcelas }(\%)\end{array}$ \\
\hline I & 63 & 77,4 \\
II & 74 & 78,0 \\
III & 24 & 23,4 \\
IV & 36 & 41,9 \\
\hline
\end{tabular}

y $16,3 \%$ palma de vino (Sheelea magdalenensis). El diámetro basal de G. ulmifolia en el potrero de estudio $(37 \mathrm{~cm}$; Cuadro 2) se encuentra dentro del rango de los datos de un estudio realizado en un potrero de Chiapas, México, donde se reporta para diámetro basal de G. ulmifolia valores entre 3 y 60 cm (López-Merlín et al., 2003).

Pithecellobium dulce, Ficus sp. y P. julioflora fueron en las que se encontraron individuos con mayor área de copa $\left(344,2 ; 339,3\right.$ y $203,5 \mathrm{~m}^{2} /$ individuo, respectivamente), siendo las que proporcionan más sombra en el área del potrero. Algunos estudios reportan para Ficus sp. un diámetro de copa promedio de hasta $25 \mathrm{~m}$ (Herrera, 2009), valor dentro del cual se encuentra la especie en este estudio $(20,0$ y $21,6 \mathrm{~cm})$. El área de copa obtenida de un individuo de la especie $P$. juliflora $\left(243 \mathrm{~m}^{2}\right)$ del potrero en estudio difiere de manera muy marcada de lo reportado por Sánchez et al. (2004), quién encontró de 22 y $45 \mathrm{~m}^{2}$ para la misma especie, en un estudio realizado en el estado Falcón, Venezuela.

Las especies con mayor índice de valor de importancia (IVI) presentaron valores medios y altos en área de copa, tal como G. ulmifolia (149,9 $\mathrm{m}^{2}$ /individuo), A. graveolens $\left(103,1 \mathrm{~m}^{2} /\right.$ individuo) y $C$. bogotense (90,8 $\mathrm{m}^{2} /$ individuo) (Figura 4).

Cojoba arborea, Senegalia affinis, Pithecellobium dulce, Triplaris americana, Ficus sp., Sheelea magdalenensis y Pseudosamanea guachapele fueron las especies con mayor altura total y de fuste promedio $(19,2$ y $7,4 \mathrm{~m}$; 18,6 y $4,3 \mathrm{~m} ; 16,6$ y $2,6 \mathrm{~m} ; 16,4$ y $6,8 \mathrm{~m} ; 14,4$ y $2,5 \mathrm{~m}$; 14,3 y 6,9 m y 12,7 y 4,1 m, respectivamente; Figura 5).

\section{Oclusión de copas}

Especies leñosas perennes con alta oclusión de su copa y apreciadas por los ganaderos fueron Crescentia cujete, Anagris foetida, Ocotea amazonica, Casearea corymbosa y Guazuma ulmifolia con 96, 92, 91, 87 y

Cuadro 2. Diámetro de troncos (diámetro a la altura del pecho-DAP y diámetro a la base- DB) promedio e índice de valor de importancia (IVI) de las especies arbóreas más importantes encontradas en un potrero del Magdalena Tolimense, Colombia. 2013.

\begin{tabular}{lccccc}
\hline \multirow{2}{*}{ Especie } & \multicolumn{2}{c}{ Db $(\mathbf{c m})$} & \multicolumn{2}{c}{ Dap $(\mathbf{c m})$} & \multirow{2}{*}{$\begin{array}{c}\text { IVI } \\
(\%)\end{array}$} \\
\cline { 2 - 5 } & Media & DE & Media & DE & $(\%, 7,7$ \\
\hline Guácimo (Guazuma ulmifolia) & 37,4 & 25,0 & 34,7 & 22,5 & 93,7 \\
Diomate (Astronium graveolens) & 29,5 & 16,5 & 27,3 & 15,4 & 69,0 \\
Angarillo (Chloroleucon bogotense) & 25,0 & 23,3 & 38,0 & 36,5 & 60,2 \\
Baho (Platymiscium hebestachyum) & 22,6 & 14,5 & 23,1 & 8,1 & 50,4 \\
Palma de vino (Sheelea magdalenensis) & 51,0 & 25,8 & 59,7 & 14,9 & 38,7 \\
Limón de cerca (Swinglia glutinosa) & 38,6 & 26,1 & 41,4 & 35,1 & 16,3 \\
Dormilón (Cojoba arborea) & 45,1 & 16,4 & 38,8 & 15,6 & 12,9 \\
Ondequera (Casearea corymbosa) & 22,7 & 12,1 & 21,0 & 7,2 & 8,9 \\
Tachuelo (Zanthoxylum caribaeum) & 24,8 & 4,8 & 20,6 & 14,9 & 8,5 \\
Varasanta (Triplaris americana) & 21,3 & 4,6 & 17,5 & 4,9 & 7,9 \\
\hline
\end{tabular}

Db: diámetro a la base, Dap: diámetro a la altura del pecho; IVI: índice de valor de importancia. 


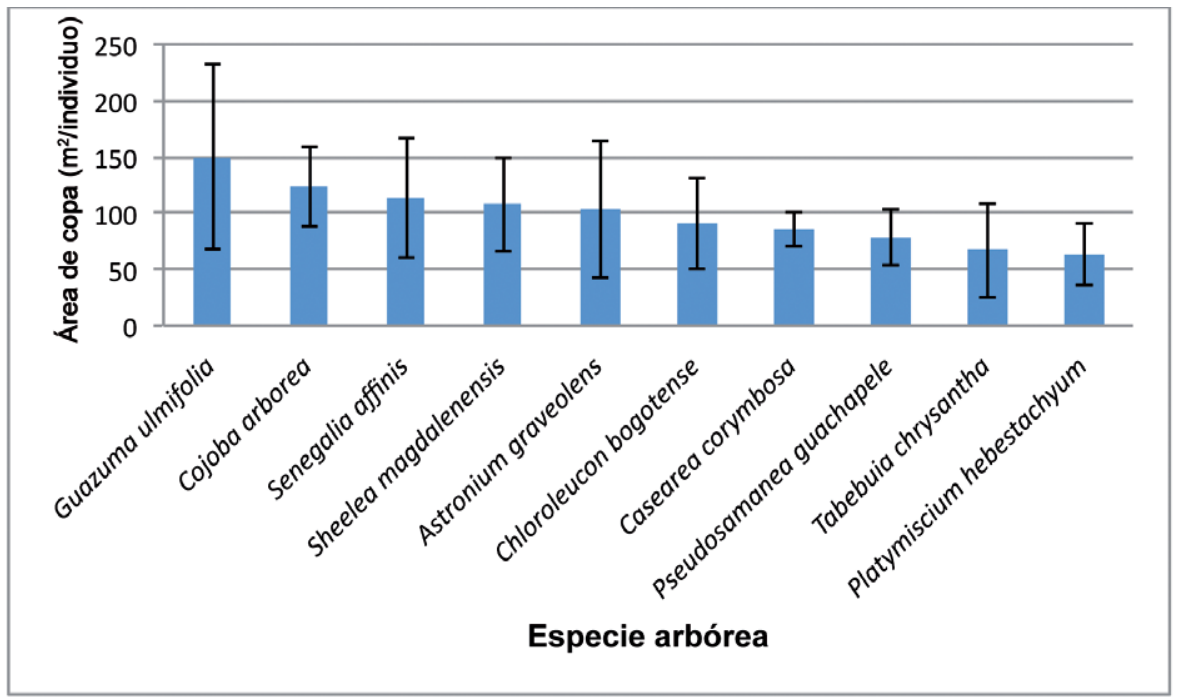

Figura 4. Área de copa promedio de especies leñosas encontradas en un potrero del Magdalena Tolimense, Colombia. 2013.

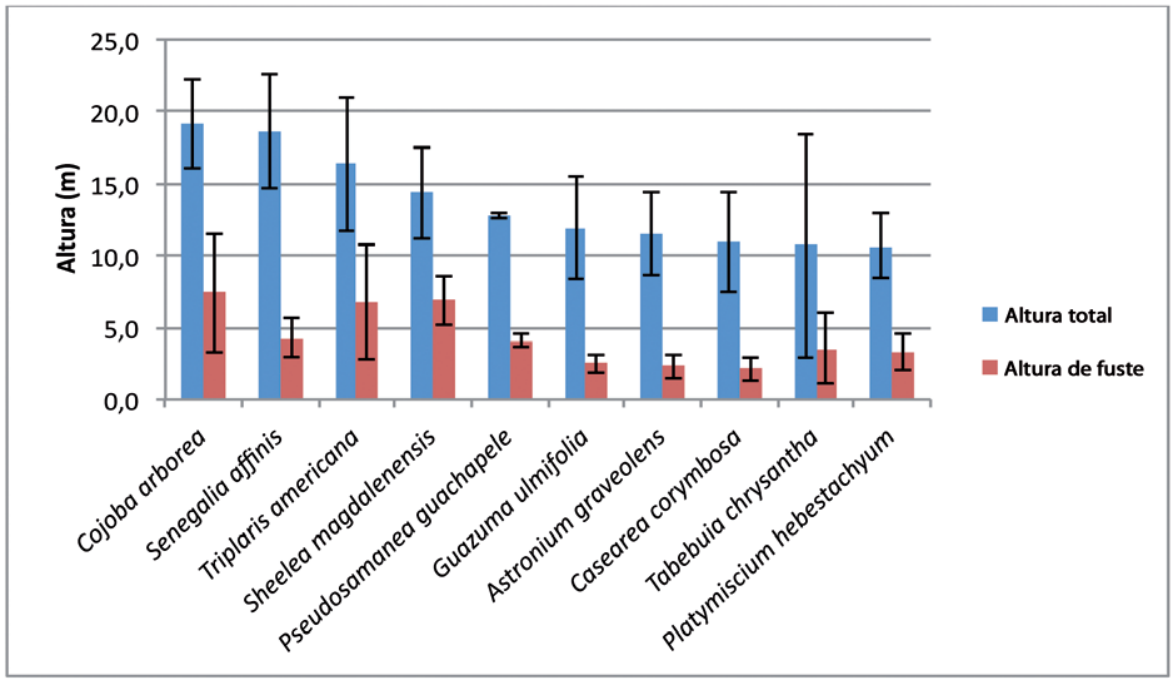

Figura 5. Altura total y de fuste promedio de especies leñosas de un potrero del Magdalena Tolimense, Colombia. 2013.

$79 \%$, respectivamente (Figura 6). Estos altos valores de oclusión de copas implica que se ejerce una alta interferencia al paso de la RAFA, insumo básico para el buen desarrollo del estrato herbáceo existente bajo el dosel. Alonso et al. (2006) reportaron que Leucaena leucocephala presentó una obstrucción del 42 a 56\% de la RAFA a una pastura de Panicum maximum en La Habana, Cuba. Estos valores están muy por debajo de lo encontrado en este estudio.

En contraste, las especies de menor oclusión de copa, es decir, aquellas que generan una sombra más rala a la pastura fueron Astronium graveolens, 


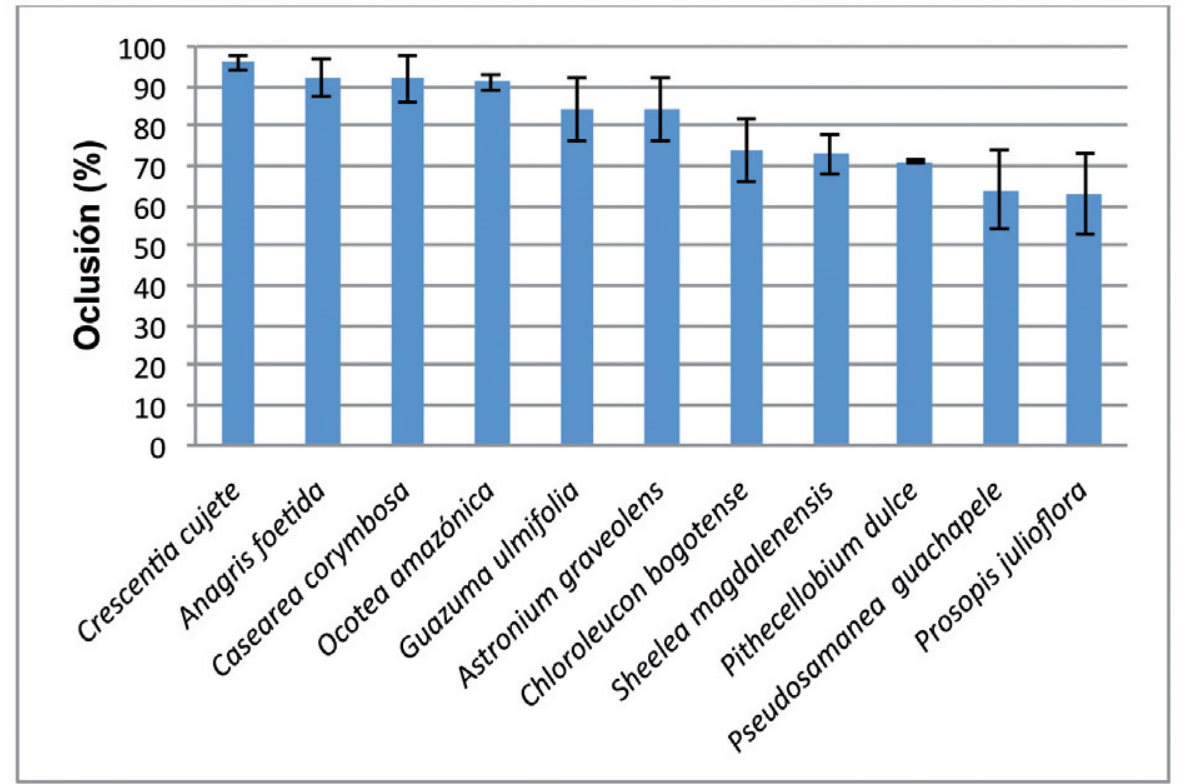

Figura 6. Oclusión de copas de las especies leñosas perennes encontradas en un potrero del Magdalena Tolimense, Colombia. 2013.

Pseudosamanea guachapele, Sheelea magdalenensis, Pithecellobium dulce y Prosopis juliflora con 74, 74, 73,71 y $63 \%$, respectivamente (Figura 6). Estos datos sugieren que en el momento de establecer o mantener especies arbóreas en las pasturas se debe tener en cuenta su efecto de interferencia de luz para fotosíntesis al estrato herbáceo, originado por las copas y la densidad de la especie que impacta la competitividad por agua y nutrientes del suelo (Daccarett y Blydenstein, 1968). Lo anterior permite direccionar los diseños de sistemas silvopastoriles hacia modelos que involucren especies leñosas con valores bajos para oclusión de copas, facilitando de esta manera el buen desarrollo de las especies herbáceas presentes bajo el dosel a partir de buena disponibilidad de luz solar.

\section{Transmisión de radiación fotosintéticamente activa por las copas de leñosas perennes}

Se encontraron especies con arquitectura de copa que permite un alto porcentaje de transmisión de RAFA, dentro de estas encontramos a $C$. arborea, $P$. juliflora, A. foetida, Z. caribaeume, $P$. guachapele con un $57,48,47,42$ y $40 \%$, respectivamente. Otro grupo de especies presenta altos niveles de intercepción de
RAFA, permitiendo que solo una pequeña fracción de esta llegue hasta la pastura, tal como G. ulmifolia (21\%), A. graveolens (18\%), O. amazonica (14\%), Swinglia glutinosa (8\%) y Ficus sp. (6\%) (Figura 7). La variabilidad en la transmisión de RAFA entre especies leñosas perennes se puede explicar por diferencias en el índice de área foliar, ángulo de inserción de las hojas, la disposición de las hojas en el tallo y el tamaño, producción y renovación de las hojas (Campbell y Grime, 1989; Kikuzawa, 1995; Berendse et al., 1999; Kikuzawa, 2003; Valladares y Brittes, 2004).

El coeficiente de correlación de Pearson para medir la relación entre el área de copa y RAFA transmitida, indicó que entre las variables estudiadas se presentó una pobre e inversa relación $(\mathrm{r}=-0,24 ; \mathrm{p}=$ $0,04)$. Es decir, cuando el área de copa estimada para el potrero en estudio fue mayor, el porcentaje de RAFA transmitido es menor (Cuadro 3).

Los valores de RAFA transmitida para algunas especies es alto en comparación con las demás. Cabe destacar especies como dormilón (Pentaclethra macroloba) (57\%), cují (Prosopis julioflora) (48\%), algarrobo hediondo (Anagris foetida) (47\%). Se observó similar tendencia en los valores de oclusión: totumo (Crescentia cujete) (96\%), algarrobo hediondo 


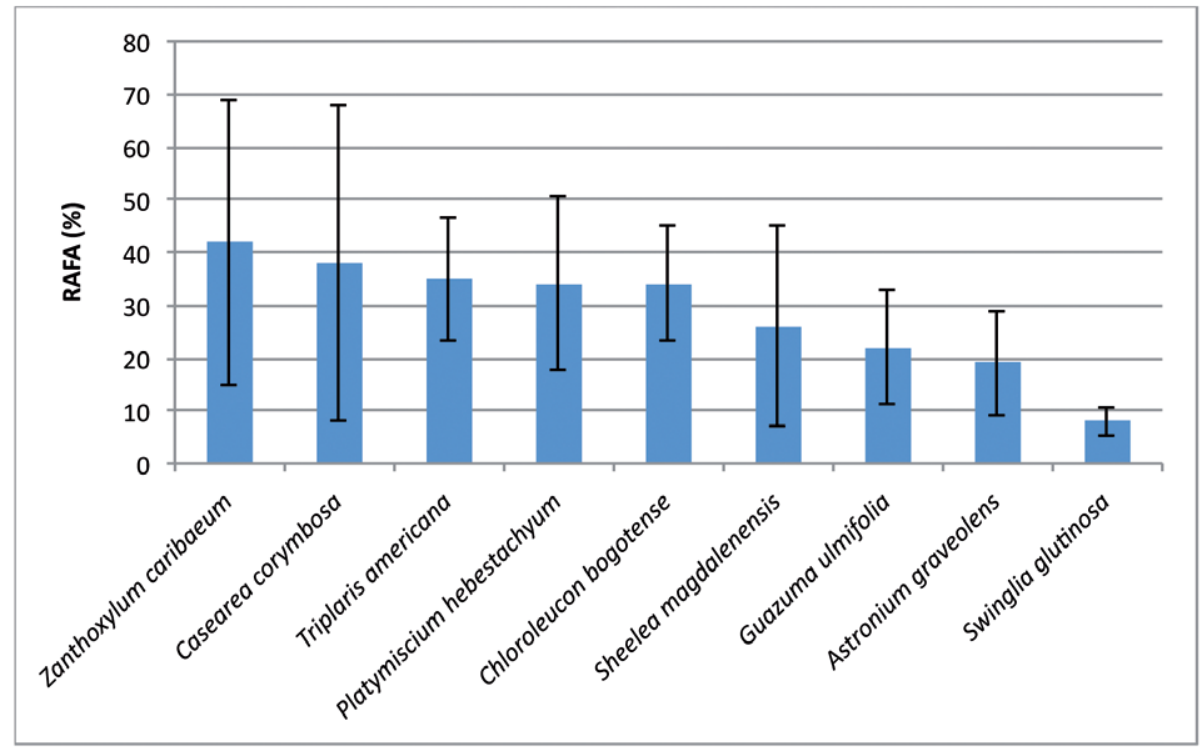

Figura 7. Porcentaje de RAFA transmitida por la copa de especies arbóreas de un potrero del Magdalena Tolimense, Colombia. 2013.

Cuadro 3. Valores para la relación área basal: área de sombra (Ab/As) de especies arbóreas en una pastura arbolada del Magdalena Tolimense, Colombia. 2013.

\begin{tabular}{|c|c|c|c|c|c|}
\hline Especie & $\begin{array}{c}\text { Área basal } \\
\left(\mathrm{m}^{2} / \text { individuo }\right)\end{array}$ & Oclusión (\%) & $\begin{array}{c}\text { Área de copa } \\
\text { (m²/individuo) }\end{array}$ & $\begin{array}{l}\text { Área de sombra } \\
\text { (m²/individuo) }\end{array}$ & $\begin{array}{c}\text { Índice } \\
(\mathrm{Ab} / \mathrm{As}) * 100\end{array}$ \\
\hline Angarillo (Chloroleucon bogotense) & 2,9 & 74 & 90,8 & 67 & 4,4 \\
\hline Baho (Platymiscium hebestachyum) & 0,8 & 80 & 64,1 & 51 & 1,6 \\
\hline Bayo (Senegalia affinis) & 0,2 & 79 & 113,6 & 90 & 0,2 \\
\hline Caucho (Ficus sp.) & 1,2 & 84 & 339,3 & 285 & 0,4 \\
\hline Chicala (Tabebuia chrysantha) & 0,2 & 92 & 67,3 & 62 & 0,2 \\
\hline Chipuelo (Anagris foetida) & 0,1 & 92 & 105,4 & 97 & 0,1 \\
\hline Cuji (Prosopis julioflora) & 0,1 & 63 & 203,5 & 128 & 0,1 \\
\hline Diomate (Astronium graveolens) & 1,3 & 84 & 103,1 & 87 & 1,5 \\
\hline Dormilon (Cojoba arborea) & 0,4 & 85 & 123,2 & 105 & 0,4 \\
\hline Guacharaco (Cupania americana) & 0,1 & 79 & 59,2 & 47 & 0,2 \\
\hline Guasimo (Guazuma ulmifolia) & 3,2 & 84 & 149,9 & 126 & 2,5 \\
\hline Igua (Pseudosamanea guachapele) & 0,1 & 64 & 78,2 & 50 & 0,2 \\
\hline Limón de cerca (Swinglia glutinosa) & 0,8 & 89 & 67,8 & 60 & 1,4 \\
\hline Mortesino (Ocotea amazonica) & 0,1 & 91 & 37,6 & 34 & 0,4 \\
\hline Ondequera (Casearea corymbosa) & 0,1 & 92 & 85,5 & 79 & 0,1 \\
\hline Palma de vino (Sheelea magdalenensis) & 2,1 & 73 & 107,8 & 79 & 2,6 \\
\hline Payande (Pithecellobium dulce) & 1,1 & 71 & 344,2 & 244 & 0,4 \\
\hline Tachuelo (Zanthoxylum caribaeum) & 0,2 & 75 & 46,7 & 35 & 0,5 \\
\hline Totumo (Crescentia cujete) & 0,0 & 96 & 43,0 & 41 & 0,1 \\
\hline
\end{tabular}


(Anagris foetida) (92\%), ondequera (Casearea corymbosa) (92\%), chicalá (Tabebuia chrysantha) (92\%) y mortesino (Ocotea amazónica) $(91 \%)$.

De acuerdo con Andrade et al. (2010), P. juliflora presenta las mejores condiciones del dosel para su inclusión en sistemas silvopastoriles, ya que tiene una alta transmisión de RAFA (48\%). Otras especies leñosas como Z. caribaeum y P. guachapele también tienen gran potencial para plantarse en potreros, debido a su alta transmisión de RAFA unido a una baja área de copa (42,1 y 40,8\% de RAFA transmitida y 44,4 y 66 $\mathrm{m}^{2} /$ árbol de área de copa, respectivamente).

Se encontró una alta diversidad de especies leñosas perennes que tiene potencial para ser introducidas a pasturas debido a la poca sombra que generan al estrato herbáceo, propiciando condiciones más favorables para la fotosíntesis.

En el diseño e implementación de sistemas silvopastoriles en estas condiciones se debería tener en cuenta las especies arbóreas que presentan características de arquitectura de dosel favorables como baja oclusión y alta RAFA transmitida, mejorando las condiciones necesarias para el proceso fotosintético de las pasturas.

\section{Relación entre el área basal y el área de sombra}

Las especies arbóreas que presentaron mayor valor de la relación área basal:área de sombra (Ab/As) fueron Chloroleucon bogotense, Sheelea magdalenensis, Guazuma ulmifolia, Astronium graveolens, Swinglia glutinosa y Platymiscium hebestachyum (Cuadro 3).

Las especies encontradas en el sitio de estudio presentan valores promedio de $\mathrm{Ab} / \mathrm{As}$ de 10,6. Este valor se aproxima a lo reportado por Andrade et al. (2012) en un estudio realizado en rastrojos de fincas ganaderas en el departamento de Caquetá, Colombia, en el cual calificaron como bajo aquellos valores para $\mathrm{Ab} / \mathrm{As}$ de especies arbóreas que presentaron en promedio 11. Estos resultados indican que las especies predominantes en el área de estudio presentan baja área basal y alta área de sombra. Sin embargo, se resalta la importancia que especies como Guazuma ulmifolia y Astronium graveolens predominen en el área, debido al aporte como suplemento en la dieta de rumiantes, generadores de confort por las condiciones micro climáticas favorables bajo su dosel y fuente de ingreso adicional a partir del aprovechamiento como maderables. Astronium graveolens se califica como una especie que provee madera de excelente calidad y fácil manejo para el acabado (Jiménez, 1986).

Se recomienda implementar estudios que puedan potenciar los valores bajos de oclusión para algunas especies arbóreas (Chloroleucon bogotense, Sheelea magdalenensis, Pithecellobium dulce, Pseudosamanea guachapele y Prosopis julioflora). Igualmente, es importante considerar para el diseño de sistemas silvopastoriles especies con alta RAFA transmitida $(P$. julioflora, Z. caribaeume, $P$. guachapele), buscando ampliar la disponibilidad de especies leñosas con características deseables en su arquitectura de copa.

En virtud de la similitud con estudios realizados en otras zonas de bosque seco tropical en América, los resultados de estos constituyen una ilustración de la estructura y función del componente leñoso en ganaderías tropicales: esto significa que mediante la realización de estudios específicos, los resultados podrían confirmarse en otras áreas de silvopasturas del neotrópico.

\section{AGRADECIMIENTOS}

Al Comité Central de Investigaciones y Desarrollo Científico de la Universidad del Tolima por el financiamiento obtenido para el desarrollo de este proyecto. A Vladimir Tobar, Mónica Pinzón y a la Organización Pajonales S.A. por el apoyo logístico.

\section{LITERATURA CITADA}

Alonso, J., G. Febles, T.E. Ruiz, y G. Achang. 2006. Efecto de la sombra en la gramínea asociada en un sistema silvopastoril de leucaena-guinea durante sus diferentes etapas. Revista Cubana de Ciencia Agrícola 40(4):503-511.

Andrade, H.J., R. Brook, y M. Ibrahim, M. 2008. Growth, production and carbón sequestration of silvopastoral systems with native timber species in the dry lowlands of Costa Rica. Plant Soil 308:11-22.

Andrade, H., R. Serrano, y M. Pinzón, M. 2010. Estructura, composición florística y características de copa del dosel de sombra en un sistema silvopastoril de árboles dispersos en potreros del Valle Cálido del Magdalena, Tolima, Colombia. En: Memorias, VI Congreso Latinoamericano Agroforestería para la Producción Pecuaria Sostenible. Serie técnica No. 15. CATIE, Turrialba. $160 \mathrm{p}$. 
Andrade, H.J., J.A. Orjuela, y A.I. Díaz. 2012. Especies regeneradas naturalmente con potencialidad de uso como árboles dispersos en pasturas en el departamento del Caquetá, Colombia. En: Memorias VII Congreso latinoamericano de sistemas agroforestales para la producción pecuaria sustentable. Belem, Brasil. p. 607-611.

Arends, E., D. Sánchez, A. Villarreal, J. Serrano, y M.E. Benítez. 2005. Bosque universitario El Caimital y la Estación Silvicultural El Manguito: dos lotes boscosos de los Llanos Occidentales para la conservación in situ de especies en peligro. Rev. For. Lat. 38:1-19.

Bárcenas-Pazos, G. 1995. Caracterización tecnológica de veinte especies maderables de la selva Lacandona. Madera y Bosques 1(1):9-38.

Bellow, J.G. 2000. Canopy variation in overstory species and assessment of light availability for shaded crops in agroforestry systems. Thesis Mag Sc, University of Florida, Florida, US.

Belsky, A.J., S.M. Mwonga, y J.M. Duxbury. 1993. Effects of widely spaced trees and livestock grazing on understory environments in tropical savannahs. Agroforestry systems 24:1-20.

Berendse, F., H. de Kroon, y W.G. Braakhekke. 1999. Acquisition, use, and loss of nutrients. En: F.I. Pugnaire, y F. Valladares, editores, Handbook of functional plant ecology. Marcel Dekker Inc., New York, Estados Unidos, p. 315-346.

Campbell, B.D., y J.P. Grime. 1989. A comparative study of plant responsiveness to the duration of episodes of mineral nutrient enrichment. New Phytologist 112: 261-267.

Chamorro, D., J. Gallo, J.C. Arcos, y M. Vanegas. 1998. Gramíneas y leguminosas, consideraciones agrozootécnicas para ganaderías de trópico bajo. Boletín de investigación. CORPOICA, Tolima, Colombia.

Daccarett, M., y J. Blydenstein. 1968. La influencia de árboles leguminosos y no leguminosos, sobre el forraje que crece bajo ellos. Turrialba 18(4):405-408.

Esquivel, H., M. Ibrahim, C.A. Harvey, C. Villanueva, T. Benjamin, y F.L. Sinclair. 2003. Árboles dispersos en potreros de fincas ganaderas en un ecosistema seco de Costa Rica. Agroforestería en las Américas 10(3940):24-29.

Frazer, G.W., C.D. Canham, y K.P. Lertzman. 1999. Gap Light Analyzer (GLA), Version 2.0. Imaging software to extract canopy structure and gap light transmission indices from true-color fisheye photographs, user's manual and program documentation. Simon Fraser University, Burnaby, British Colombia, and the Institute of Ecosystem Studies, Millbrook, New York, USA.

González-Hernández, M.P., y M.J. Rozados-Lorenzo. 2008. Pasture production and tree growth in agroforetry systems of Northwest Spain. En: D.R. Batish, R.K. Kohli, S. Jose, y H.P. Singh, editores, Ecological basis of agroforestry. Taylor and Francis Group, LLC, Boca Raton, Florida, USA. p. 361-376.

Jiménez, Q. 1986. El ron-ron (Astronium graveolens Jacq.). Biocenosis 3(2):14-16.

Harvey, C.A., C. Villanueva, J. Villacis, M. Chacón, D. Muñoz, M. López, M. Ibrahim, R. Gómez, R. Taylor, J. Martínez, A. Navas, J. Sáenz, D. Sánchez, A. Medina, S. Vílchez, B. Hernández, A. Pérez, E. Ruiz, F. López, I. Lang, y F.L. Sinclair. 2005. Contribution of live fences to the ecological integrity of agricultural landscapes. Agriculture Ecosystems \& Environment 111:200-230.

Harvey, C.A., y W.A. Haber. 1999. Remnant trees and conservation of biodiversity in Costa Rican pastures. Agroforestry Systems 44:37-68.

Herrera, S. 2009. Árboles de la Universidad del Valle. Universidad del Valle, Cali, Colombia.

Holdridge, L.R. 1967. Ecología basada en zonas de vida. Trad. Humberto Jiménez Saa. IICA, San José, Costa Rica.

IAVH (Instituto Alexander von Humboldt). 1998. El bosque seco tropical en Colombia. Programa de Inventario de la Biodiversidad Grupo de Exploraciones y Monitoreo Ambiental GEMA, Colombia

Kaimowitz, D., y A. Angelsen. 2008 Will livestock intensification help save Latin America's forests? Journal of Sustainable Forestry 27(1-2):6-24.

Kay, S., P. Spruyt, y K. Alexandrou. 2003. Geometric quality assessment of orthorectified VHR space image data. Photogrammetric Engineering \& Remote Sensing 69:484-491.

Kikuzawa, K. 1995. Leaf phenology as an optimal strategy for carbon gain in plants. Canadian Journal of Botany 73:158-163.

Kikuzawa, K. 2003. Phenological and morphological adaptations to the light environment in two woody and two herbaceous plant species. Functional Ecology 17:29-38

Lamprecht, H. 1990. Silvicultura en los trópicos. Trad. A. Cacillo. GTZ, Eschborn, Alemania.

López-Merlín, D., L. Soto-Pinto, G. Jiménez-Ferrer, y S. Hernández-Daumas. 2003. Relaciones alométricas para 
la producción de biomasa forrajera y leña de Acacia pennatula y Guazuma ulmifolia en dos comunidades del norte de Chiapas México. Interciencia 28(6):334-339.

Manríquez-Mendoza, L.Y., S. López-Ortíz, P. PérezHernández, E. Ortega-Jiménez, Z.G. López-Tecpoyotl, y M. Villarruel-Fuentes. 2011. Agronomic and forage characteristics of Guazuma ulmifolia Lam. Tropical and Subtropical Agroecosystems 14(2):453-463.

Ospina, A. 2003. Agroforestería aportes conceptuales, metodológicos y prácticos para el estudio agroforestal. Asociación del Colectivo del Suroccidente Colombiano, Cali, Colombia.

Restrepo, C. 2002. Relaciones entre la cobertura arbórea en potreros y la producción bovina en fincas ganaderas en el trópico seco, Cañas, Costa Rica. M.Sc. Tesis, CATIE, Turrialba, Costa Rica

Sánchez, A.J., F.A. Pariacote, S. Alfonzo, y R. Flores. 2004. Arquitectura y fenología de las especies Prosopis juliflora y Acacia tortuosa en el semiárido del Estado Falcón, Venezuela. Archivos Latinoamericanos de Producción Animal 12(4 sup. 1):72-81.

Souza de Abreu, M., M. Ibrahim, C. Harvey, y F. Jiménez. 2000. Caracterización del componente arbóreo en los sistemas ganaderos de la Fortuna de San Carlos,
Costa Rica. Agroforestería en las Américas 7(26):5356.

Souza de Abreu, M.H. 2002. Contribution of trees to the control of heat stress in dairy cows and the financial viability of livestock farms in humid tropics. PhD. Thesis, CATIE, Turrialba, Costa Rica.

Szott, L., M. Ibrahim, y J. Beer. 2000. The hamburger connection hangover: cattle, pasture land degradation and alternative land use in Central America. CATIEDANIDA-GTZ, Turrialba, Costa Rica. 71 p.

Valladares, F., y D. Brittes. 2004. Leaf phyllotaxis: does it really affect light capture? Plant Ecology 174:11-17.

Zamora, S., J. Garcia, G. Bonilla, H. Aguilar, C.A. Harvey, y M. Ibrahim. 2001. Como utilizar los frutos de Guanacaste (Enterolobium cyclocarpum), guácimo (Guazuma ulmifolia), genizaro (Pithecellobium saman) y jicaro (Crescentia alata) en alimentación animal. Agroforestería en las Américas 8:45-49.

Zapata-Arango, P.C. 2010. Efecto del guácimo (Guazuma ulmifolia), carao (Cassia grandis) y roble (Tabebuia rosea) sobre la productividad primaria neta área y composición florística de pasturas naturales. Muy Muy y Matiguás, Nicaragua. Tesis M.Sc., CATIE, Turrialba, Costa Rica. 